\title{
R\&D Spillovers in an Endogenous Growth Model with Physical Capital, Human Capital and Varieties
}

\author{
Tiago Neves Sequeira* \\ Accepted for publication in Macroeconomic Dynamics
}

\begin{abstract}
There is a family of models with Physical, Human capital and R\&D for which convergence properties have been discussed (Arnold, 2000a; Gómez, 2005). However, spillovers in R\&D have been ignored in this context. We introduce spillovers in this model and derive its steady-state and stability properties. This new feature implies that the model is characterized by a system of four differential equations. A unique Balanced Growth Path along with a two dimensional stable manifold are obtained under simple and reasonable conditions. Transition is oscillatory toward the steady-state for plausible values of parameters.
\end{abstract}

JEL Classification: C62, O11, O41.

Key-Words: convergence; stability; spillovers; R\&D.

*Departamento de Gestão e Economia, Universidade da Beira Interior and INOVA research center, Faculdade de Economia, Universidade Nova de Lisboa. (e-mail: sequeira@ubi.pt). I thank Robert Lucas, Ana Balcão Reis, participants in the ASSET 2007 conference in Padova (Italy) and in the fist conference of the Portuguese Economic Journal in Azores (Portugal) for useful comments. I gratefully acknowledge financial support from POCI/FCT - Fundação para a Ciência e Tecnologia. The usual disclaimer applies. This paper has been accepted for publication and will appear in revised form in Macroeconomic Dynamics, published by Cambridge University $\operatorname{Press}^{R}$. 


\section{Introduction}

Arnold (1998, 2000a) introduced a model with Physical Capital, Human Capital and R\&D and studied its convergence properties, without considering spillovers in the R\&D technology. Funke and Strulik (2000) integrated three models as different stages of economic development and presented a model with Physical Capital, Human Capital and R\&D similar to that in Arnold (1998) as a developed country stage. Gómez (2005) showed that the convergence features of this model change dramatically when accounting for the predetermination of $r$, and has fully characterized the model convergence properties and derived stability conditions. In particular, the model converges through a two-dimensional stable manifold with oscillatory dynamics. Before these contributions, human capital-based growth models and R\&D-based growth models were studied separately. Benhabib and Perli (1994) showed that an endogenous growth model with externalities in human capital accumulation yields indeterminacy of equilibria. Ladrón-deGuevara et al. (1997) studied the equilibrium dynamics of two extensions of the Uzawa-Lucas framework, in which they discovered multiple equilibrium in a model with leisure, but without externalities. Arnold (2000b,c) demonstrated that the steady-state of the Romer (1990) model of R\&D is globally saddle-point stable. Eicher and Turnovsky (2001) showed that a two-sector R\&D-based non-scale growth model is represented by a two-dimensional stable saddle-path with oscillatory transitional dynamics. Arnold (2006) studied the stability properties of the Jones (1995) model and concluded for a unique Balanced Growth Path on a two-dimensional stable manifold which show monotonic transition for a broad range of parameters. These last two articles showed that the system of differential equations to be analyzed in non-scale R\&D growth models is of order four.

We add spillovers in R\&D to the Arnold (2000a) model and derive the model equilibrium and stability properties. It is worth noting that the existence and high magnitude of spillovers have been empirically proved by recent literature (e.g. Grilliches, 1992; Porter and Stern, 2000). Engelbrecht (1997) and Barrio-Castro (2002) concluded for the existence of statistically significant $R \& D$ spillovers in empirical specifications that included human capital but models that jointly consider human capital accumulation and R\&D have not taken them into account until now. Additionally to derive the steady-state and its stability properties, we numerically solve our model for the transition path.

The consideration of spillovers in the model provides a unique Balanced 
Growth Path. In comparison with the Arnold (1998, 2000a) model, whose stability properties were studied in Gómez (2005), the consideration of spillovers increases the order of the differential equations system to four (as in the non-scale R\&D growth models). Because of that this article also constitutes an example of the analysis of high-dimensional systems of ODEs in economics and of the possible technical tools to use. In terms of results, it maintains the oscillatory convergence for typical calibrations. It is also shown that this model allows for a lower and more reasonable value for the markup to fulfill the stability conditions in comparison to what happened in Gómez (2005).

In comparison with Jones' model, whose stability properties have been studied in Arnold (2006), the introduction of accumulation of human capital maintains the unique trajectory to the steady-state. In contrast to what happened in the Jones model, we show that this model presents oscillatory convergence for typical and broad parameters values.

In Section 2, we present the model. In Section 3, we present the Balanced Growth Equilibrium and the stability analysis of the steady-state. In Section 4, we calibrate the model, give some examples and solve the model numerically. At the end of this section, we discuss the results. In section 5, we conclude.

\section{The Model}

This section recapitulates the Arnold (1998, 2000a) model, assuming a different function for the production of new ideas, which accounts for the existence of spillovers in R\&D.

\subsection{Setup of the Model}

Consider a closed economy inhabited by a constant population, normalized to one, of identical infinitely-lived households that maximize the intertemporal utility function:

$$
\int_{0}^{\infty} \frac{C^{1-\theta}}{1-\theta} e^{-\rho t} d t, \rho>0, \theta>0,
$$

(where $C$ denotes consumption, $\rho$ is the time-discount rate and $\theta$ is the relative risk aversion coefficient), subject to the budget constraint and the 
knowledge accumulation technology. Human Capital, $H$, can be devoted to production $\left(H_{Y}\right)$, education $\left(H_{H}\right)$ and $\mathrm{R} \& \mathrm{D}\left(H_{n}\right)$, respectively:

$$
H=H_{Y}+H_{H}+H_{n}
$$

and is calculated according to:

$$
\dot{H}=\xi H_{H}
$$

The accumulation of human capital is a non-market activity. R\&D technology is equal to that in Jones (1995), with no duplication effects in human capital, given by

$$
\dot{n}=\epsilon H_{n} n^{\phi},
$$

where $0<\phi<1$ is the parameter that governs spillovers and $n$ is the number of available varieties. $\phi>0$ implies that to some extent the development of new varieties depends on the stock of previous available varieties: the "stand on the shoulders" effect. With $\phi=0$, this would be the Arnold model described in Gómez (2005). The R\&D process is possible because of monopolistic competition in differentiated goods, as we will describe later. The budget constraint faced by the household is

$$
\dot{W}=r W+w\left(H-H_{H}\right)-C .
$$

where $r$ is the return per unit of aggregate wealth, $W$, and $w$ the wage per unit of employed human capital, $H-H_{H}$. Let $g_{z}=\frac{\dot{z}}{z}$ denote the growth rate of any variable $z$. The first-order conditions for maximization of (1), using (3) and (5) as constraints give:

$$
\begin{aligned}
& g_{C}=(r-\rho) / \theta, \\
& g_{w}=r-\xi .
\end{aligned}
$$

A single homogeneous final good $Y$ is produced with Cobb-Douglas technology

$$
Y=K^{\beta} D^{\eta} H_{Y}^{1-\beta-\eta}, \beta>0, \eta>0, \beta+\eta<1,
$$

where $K$ is physical capital, $H_{Y}$ is human capital allocated to final good production and $D$ is an index of differentiated goods, 


$$
D=\left[\int_{0}^{n} x_{i}^{\alpha} d i\right]^{1 / \alpha}, 0<\alpha<1
$$

where $x_{i}$ is the amount used for each one of the $n$ intermediate goods and $\alpha$ governs the substitutivity between varieties. The market for the final good is perfectly competitive and its price is normalized to one. Profit maximization gives the following inverse factor demands:

$$
\begin{gathered}
r=\frac{\beta Y}{K}, \\
P_{D}=\frac{\eta Y}{D},
\end{gathered}
$$

and

$$
w=\frac{(1-\beta-\eta) Y}{H_{Y}},
$$

where $P_{D}$ represents the price index for intermediates.

Each firm in the differentiated-goods sector owns a patent for selling its variety $x_{i}$. Let $v_{t}$ denote the expected value of innovation, defined by

$$
v_{t}=\int_{t}^{\infty} e^{-[R(\tau)-R(t)]} \pi(\tau) d \tau, \text { where } R(t)=\int_{0}^{t} r(\tau) d \tau .
$$

Taking into account the cost of innovation as implied by (4), free entry conditions in $\mathrm{R} \& \mathrm{D}$ are defined as follows:

$$
\begin{gathered}
w / \epsilon>v n^{\phi} \text { if } \dot{n}=0\left(H_{n}=0\right) \text { or } \\
w / \epsilon=v n^{\phi} \text { if } \dot{n}>0\left(H_{n}>0\right) .
\end{gathered}
$$

where $w$ is the wage paid to human capital.

Finally, no-arbitrage requires that the valorization of the patent plus profits is equal to investing resources in the riskless asset:

$$
\dot{v}+\pi=r v \Leftrightarrow \frac{\dot{v}}{v}=r-\pi / v .
$$

Producers act under monopolistic competition and maximize operating profits 


$$
\pi_{i}=\left(P_{x_{i}}-1\right) x_{i}
$$

The variable $P_{x_{i}}$ denotes the price of an intermediate and 1 is the unit cost of $Y$. From profit maximization in the intermediate-goods sector, each firm charges a price

$$
P_{x_{i}}=1 / \alpha .
$$

With identical technologies and symmetric demand, the quantity supplied is the same for all goods, $x_{i}=x$. Hence, equation (9) simplifies to

$$
D=n^{1 / \alpha} x .
$$

From $P_{D} D=P_{x_{i}}$ xn together with equations (18) and (19) we obtain the total quantity of intermediates employed as

$$
X=x n=\alpha \eta Y .
$$

After insertion of equations (18) and (20) into (17), profits can be rewritten as a function of aggregate output and the number of existing varieties:

$$
\pi=(1-\alpha) \eta Y / n \text {. }
$$

Before we proceed with the analysis we compute some equations that will be useful. The number of varieties in the denominator of (21) indicates the business-stealing effect. Insertion of equation (20) in the resource constraint $K=Y-\int_{0}^{n} x_{i} d i-C$ simplifies it to

$$
\dot{K}=(1-\alpha \eta) Y-C
$$

and insertion of (19) and (20) in the production function (8) gives:

$$
Y^{1-\eta}=(\alpha \eta)^{\eta} K^{\beta} n^{\eta \frac{1-\alpha}{\alpha}}\left(u_{1} H\right)^{1-\beta-\eta},
$$

where $u_{1}=\frac{H_{Y}}{H}$ is the proportion of human capital employed in the final good production. Similarly, we also denote $u_{2}=\frac{H_{n}}{H}$ as the share of human capital allocated to research and $u_{3}=\frac{H_{H}}{H}$ as the share of human capital allocated to human capital accumulation. After time-differentiation of the previous function we obtain the output growth rate

$$
(1-\eta) g_{Y}=\beta g_{K}+\left[\frac{1-\alpha}{\alpha}\right] \eta g_{n}+(1-\beta-\eta)\left(g_{u_{1}}+g_{H}\right)
$$


Log-differentiation of equations (10) and (12) provides

$$
\begin{gathered}
g_{r}=g_{Y}-g_{K}, \\
g_{w}=g_{Y}-\left(g_{u_{1}}+g_{H}\right) .
\end{gathered}
$$

To avoid excessive length we will concentrate in the innovative economy description $(\dot{n}>0){ }^{1}$

\subsection{The Dynamics of the Economy}

The economy is characterized by the presence of physical capital accumulation $(\dot{K}>0)$, human capital accumulation $(\dot{H}>0)$ and R\&D $(\dot{n}>0)$. We now derive the system that describes the dynamics of the economy. From (6) and (22) and then using (10), we obtain $g_{\chi}$ :

$$
g_{\chi}=\left(\frac{1}{\theta}-\frac{1-\alpha \eta}{\beta}\right) r+\chi-\frac{\rho}{\theta}
$$

where $\chi=C / K$. Departing from (24) and using (25), (26) and then substituting $g_{w}$ by $(7)$, we obtain $g_{r}$ :

$$
g_{r}=-\frac{1-\beta-\eta}{\beta}(r-\xi)+\frac{\eta}{\beta} \frac{1-\alpha}{\alpha} g_{n},
$$

Inserting (7), (12), (15) and (21) into (16), we obtain

$$
u_{1}=\frac{(1-\beta-\eta)\left(\xi+\phi g_{n}\right)}{\epsilon(1-\alpha) \eta \psi} .
$$

Using this last equation, eq. (3) and the definition $\psi=H / n^{1-\phi}$ (noting that $u_{1}+u_{2}+u_{3}=1$ ), we obtain the growth rate of $\psi$ :

$$
g_{\psi}=\xi\left(1-\left[\frac{(1-\beta-\eta)\left(\xi+\phi g_{n}\right)}{(1-\alpha) \eta}+g_{n}\right] \frac{1}{\epsilon \psi}\right)-(1-\phi) g_{n} .
$$

\footnotetext{
${ }^{1}$ In fact, as the change we introduce is in the R\&D production process, the eventual existence of a previous stage $(\dot{n}=0)$ would not change anything in the developing economy stage in Gómez (2005) or in Funke and Strulik (2000).
} 
We note that $g_{n}$ is no longer given as a function of $\chi$ and $r$, which would be directly substituted in previous equations, as in previous contributions (e.g. Gómez, 2005:5). From $(29), g_{u_{1}}=(1-\phi) g_{n}-g_{H}+\frac{\phi \dot{g}_{n}}{\left(\xi+\phi g_{n}\right)}$. Then, we note from (26) that $g_{u_{1}}=g_{Y}-g_{w}-g_{H}$. If $\phi=0$ then $g_{u_{1}}=g_{n}-g_{H}$, as in Arnold (2000) and one could write $g_{n}=g_{r}+\frac{1-\alpha \eta}{\beta} r-\chi-(r-\xi)$ which would be substituted in (28) and (30). This would mean that the locus $\dot{g}_{n}=0$ is independent of other variables in the model and Arnold (2000) could study convergence using two separate phase diagrams. However, with $\phi>0$, this separability is lost. In this case, using (25), $g_{K}$ from (22) and (7), we obtain the equation that describes the evolution of $g_{n}$ :

$$
\dot{g_{n}}=\frac{\xi+\phi g_{n}}{\phi}\left[g_{r}+\frac{1-\alpha \eta}{\beta} r-\chi-(r-\xi)-(1-\phi) g_{n}\right] .
$$

The system composed by equations (27), (28), (30) and (31) describes the evolution of the economy. We can further simplify eq. (31), substituting $g_{r}$ from (28):

$$
\dot{g_{n}}=\frac{\xi+\phi g_{n}}{\phi}\left[\frac{(1-\alpha) \eta}{\beta} r-\chi+\frac{1-\eta}{\beta} \xi+\left(\frac{\eta}{\beta} \frac{1-\alpha}{\alpha}-(1-\phi)\right) g_{n}\right] \text {. }
$$

This is the differential equation that enters the system, when the model is compared to that in Arnold (2000).

\section{Balanced Growth Equilibrium}

In this section, we first derive equations that describe the steady-state and then we study the convergence properties around the steady-state.

\subsection{The Steady-State: existence}

In this sub-section, we present the equations that describe the steady-state of the model and demonstrate its existence.

Theorem 1 Let $\xi>\rho$ and $\theta>1$. There is one unique positive steady-state of the model given by $\left(r^{*}, \chi^{*}, \psi^{*}, g_{n}^{*}\right)$, as follows: 


$$
\begin{aligned}
r^{*} & =\frac{\theta\left(\frac{(1-\beta-\eta)}{\eta} \frac{\alpha}{1-\alpha}(1-\phi)+1\right) \xi-\rho}{\theta\left(\frac{(1-\beta-\eta)}{\eta} \frac{\alpha}{1-\alpha}(1-\phi)\right)+(\theta-1)}, \\
\chi^{*} & =\left(\frac{1-\alpha \eta}{\beta}-\frac{1}{\theta}\right) r^{*}+\frac{\rho}{\theta}, \\
\psi^{*} & =\frac{\xi\left[g_{n}^{*}(1-\alpha) \eta+(1-\beta-\eta)\left(\xi+\phi g_{n}^{*}\right)\right]}{(1-\alpha) \eta \epsilon\left(\xi-(1-\phi) g_{n}^{*}\right)}, \\
g_{n}^{*} & =\frac{r^{*}(1-\theta)+\theta \xi-\rho}{\theta(1-\phi)} .
\end{aligned}
$$

Proof. The shares of human capital to different sectors must be constant for an interior steady-state solution. In particular, the fact that the share in human capital accumulation $u_{3}^{*}$ is constant implies by (3) that $g_{H}^{*}$ is constant. With $u_{1}^{*}$ and $u_{3}^{*}$ constant, $g_{n}^{*}$ and $\psi^{*}$ must be constant, by (4) and (29). Thus $g_{H}^{*}=(1-\phi) g_{n}^{*}$. From constancy of $\psi^{*}$ and $u_{1}^{*}$ we can say that $g_{Y}^{*}=g_{K}^{*}$. This equality, equation (24) and the constancy of $g_{n}^{*}$, $g_{H}^{*}$ and $u_{1}^{*}$ imply that $r^{*}, g_{Y}^{*}$ and $g_{K}^{*}$ are constant. Thus $\chi^{*}=(C / K)^{*}$ is constant (to see this divide $(22)$ by $K$ ). We now derive necessary and sufficient conditions for positivity. For $r^{*}>0$ we reach $\left(A_{1}+1\right) \theta \xi>\rho$ and $\theta A_{1}+(\theta-1)>0$, where $A_{1}=\frac{1-\beta-\eta}{\eta} \frac{\alpha}{1-\alpha}(1-\phi)$. For $\chi^{*}>0$, we have $\left(A_{1}+1\right) \theta \xi>A_{2} \rho$, where $A_{2}=\frac{\theta\left(\frac{1-\alpha \eta}{\beta}-1\right)-A_{2}}{\left(\theta \frac{1-\alpha \eta}{\beta}-1\right)}$, which (if $\theta \geq 1$ ) is always verified for $r^{*}>0 .^{2}$ For $\psi^{*}>0$ we reach $(\theta-1)\left(A_{1}+1\right) \theta \xi+A_{1} \theta \rho>0$, using that $r^{*}>0$. Finally $g_{n}^{*}>0$ implies $\xi>\rho$ if $r^{*}>0$. This condition together with $\theta \geq 1$ simultaneously imply $r^{*}>0, \psi^{*}>0$ and $\chi^{*}>0$. These two simple conditions are sufficient for a feasible steady-state.

Positivity of $\psi^{*}$ is directly implied by the transversality condition on $H$. Transversality condition on human capital may be written as

$$
\lim _{t \rightarrow \infty} e^{-\rho t} \lambda_{2}(t) H(t)=0
$$

$\left(\lambda_{2}\right.$ is the co-state of $\left.H\right)$, which converts on $\left(-\rho+\frac{\dot{\lambda_{2}}}{\lambda_{2}}+g_{H}\right)<0$. As $\frac{\dot{\lambda_{2}}}{\lambda_{2}}=$ $\rho-\xi$ and $g_{H}^{*}=(1-\phi) g_{n}^{*}$, the transversality condition is equivalent to $\xi-(1-\phi) g_{n}^{*}>0$, which is equivalent to $(\theta-1)\left(A_{1}+1\right) \theta \xi+A_{1} \theta \rho>0$, stated above.

\footnotetext{
${ }^{2}$ By initial assumptions on parameters $1-\alpha \eta>\beta$ (see eq. 8 ). For $\theta \geq 1, A_{2}<1$.
} 


\subsection{Stability}

We will now analyze the dynamics of the model in the neighborhood of the steady-state.

The analysis of the linearized system around the steady-state will establish that for most reasonable values, the system has two eigenvalues with negative real parts. We also show that initial state conditions $K(0), H(0)$ and $n(0)$ are sufficient to determine the initial point in the two-dimensional stable manifold, thus the Balanced Growth Path is uniquely determined. However, as in Eicher and Turnovsky (2001:95), due to the complexity of the model, we cannot rule out the case of instability.

Linearizing the system (27), (28), (30) and (31) around its steady-state $\left(r^{*}, \chi^{*}, \psi^{*}, g_{n}^{*}\right)$ gives the following fourth-order system:

$$
\begin{aligned}
\left(\begin{array}{c}
\dot{r} \\
\dot{\chi} \\
\dot{\psi} \\
\dot{g_{n}}
\end{array}\right) & =\left(\begin{array}{cccc}
-\frac{1-\beta-\eta}{\beta} r^{*} & 0 & 0 & \frac{\eta}{\beta} \frac{1-\alpha}{\alpha} r^{*} \\
\left(\frac{1}{\theta}-\frac{1-\alpha \eta}{\beta}\right) \chi^{*} & \chi^{*} & 0 & 0 \\
0 & 0 & \xi-(1-\phi) g_{n}^{*} & -B_{1}-(1-\phi) \psi^{*} \\
\frac{\xi+\phi g_{n}^{*}}{\phi} \frac{(1-\alpha) \eta}{\beta} & -\frac{\xi+\phi g_{n}^{*}}{\phi} & 0 & B_{2} g_{n}^{*}-B_{3} \frac{\xi}{\phi g_{n}^{*}}
\end{array}\right)\left(\begin{array}{c}
r-r^{*} \\
\chi-\chi^{*} \\
\psi-\psi^{*} \\
g_{n}-g_{n}^{*}
\end{array}\right) \\
\text { where } B_{1} & =\xi \phi \frac{(1-\beta-\eta)}{(1-\alpha) \eta} \frac{1}{\epsilon}+\frac{\xi}{\epsilon} ; B_{2}=\left(\frac{\eta}{\beta} \frac{1-\alpha}{\alpha}-(1-\phi)\right) \\
B_{3} & =\left(\frac{(1-\alpha) \eta}{\beta} r-\chi+\frac{1-\eta}{\beta} \xi\right)
\end{aligned}
$$

or $\dot{\mathbf{X}}=\mathbf{J}\left(\mathbf{X}-\mathbf{X}^{*}\right)$, where $\mathbf{J}$ is the Jacobian in (38). To demonstrate the conditions under which the system is completely stable and unstable we state the following theorem.

Theorem 2 If a feasible steady-state exists, there are two possible trajectory solutions: there exists a unique steady-state to which a unique path converges, or the steady-state is unstable.

The proof makes use of four lemmas. The first two demonstrate that there are zero or two stable roots, i.e., the stable manifold is two dimensional or it is unstable. The third presents a sufficient condition under which the solution is the first: two stable roots. The fourth demonstrates that, when there are two stable roots, the balanced growth path is uniquely determined by the state variables in the model. 
Lemma 1 There is an even number of stable roots, zero (instability), two (stability) or four (indeterminacy).

Proof. This is implied by the fact that, for a feasible steady-state, the determinant of (38) is positive. This is,

$$
-\left[B_{2} g_{n}^{*}-B_{3} \frac{\xi}{\phi g_{n}^{*}}\right] \frac{1-\beta-\eta}{\beta}-\frac{\eta}{\beta} \frac{1-\alpha}{\alpha}\left[\frac{1}{\theta}-\frac{1-\eta}{\beta}\right] \frac{\xi+\phi g_{n}^{*}}{\phi}>0 .
$$

We provide a proof. Using the fact that, by (32), in steady-state $B_{3}=$ $-B_{2} g_{n}^{*}$, and by simplifying terms, the expression (39) turns out to be equal to the denominator of $r^{*}$, for a positive steady-state $\left(r^{*}>0, \chi^{*}>0, \psi^{*}>0\right.$, $\left.g_{n}^{*}>0\right)$ :

$$
\theta\left(\frac{(1-\beta-\eta)}{\eta} \frac{\alpha}{1-\alpha}(1-\phi)\right)+(\theta-1)>0,
$$

which implies $\theta>1$ as a sufficient condition. Thus, the system has an even number of stable roots, zero, two or four under the same conditions for a positive steady-state.

Lemma 2 There are not four stable roots.

Proof. We can rule out indeterminacy, as there is always a positive root eliminating the possibility of having four roots with negative real parts. The case with four negative roots is excluded, as there is a root $e_{3}$ :

$$
e_{3}=\xi-(1-\phi) g_{n}^{*}
$$

It was already shown that $\xi-(1-\phi) g_{n}^{*}>0$ by the transversality condition on human capital accumulation (see the Proof of Theorem 1).

Lemma 3 A sufficient condition to rule out the instability outcome is $\frac{1}{\alpha}<$ $1+\frac{(1-\beta-\eta)}{\eta}$.

Proof. As by Lemmas 1 and 2 we remain with the possibility of zero or two stable roots, we only need to discover a sufficient condition for the existence of one stable root. By Lemmas 1 and 2, this is also a sufficient condition to obtain two stable roots, which guarantees stability. We use the Gershgorin Disc Theorem (e.g. Horn and Johnson, 1985) to determine this sufficient condition. Applying Corollary 1 of the Disc Theorem to matrix 
$J$ in (38), we can see that for any positive real numbers $d_{1}, d_{2}, d_{3}, d_{4}$ the eigenvalues of $J e_{1}, \ldots, e_{4}$ are contained in the discs:

$$
\begin{gathered}
\left|z-\left(-\frac{1-\beta-\eta}{\beta} r^{*}\right)\right| \leq \frac{d_{4}}{d_{1}}\left|\frac{\eta}{\beta} \frac{1-\alpha}{\alpha} r^{*}\right| \\
\left|z-\chi^{*}\right| \leq \frac{d_{1}}{d_{2}}\left|\left(\frac{1}{\theta}-\frac{1-\alpha \eta}{\beta}\right) \chi^{*}\right| \\
\left|z-\left(\xi-(1-\phi) g_{n}^{*}\right)\right| \leq \frac{d_{4}}{d_{3}}\left|-B_{1}-(1-\phi) \psi^{*}\right| \\
\left|z-\left(B_{2} g_{n}^{*}-B_{3} \frac{\xi}{\phi g_{n}^{*}}\right)\right| \leq \frac{d_{1}}{d_{4}}\left|\frac{\xi+\phi g_{n}^{*}}{\phi} \frac{(1-\alpha) \eta}{\beta}\right|+\frac{d_{2}}{d_{4}}\left|-\frac{\xi+\phi g_{n}^{*}}{\phi}\right|
\end{gathered}
$$

where $|\cdot|$ denotes the absolute value. Let $d_{1}=d_{2}=d_{4}=1 .^{3}$ In order to obtain a sufficient condition to stability, we only need to look at the first disc with center in $-\frac{1-\beta-\eta}{\beta} r^{*}$ and radius $\frac{\eta}{\beta} \frac{1-\alpha}{\alpha} r^{*}$ and prove that it is all contained in the left half of the complex plane, so that there exists a negative eigenvalue within that circle. Signing the terms in (42) we obtain the sufficient condition:

$$
-\frac{1-\beta-\eta}{\beta}+\frac{\eta}{\beta} \frac{1-\alpha}{\alpha}<0
$$

which is easily converted into the meaningful expression in the Lemma's text. ${ }^{4}$

The conditions stated in Theorem 2 and in its lemmas are verified for a broad range of parameters and particularly for sufficiently low markups, as indicated by (46).

Lemma 4 If the system of four differential equations has two stable roots, the state variables $K(0), H(0)$ and $n(0)$ uniquely determine the starting point in the stable manifold.

\footnotetext{
${ }^{3}$ The eigenvalue $e_{3}$ stated in Lemma 2 can be recovered using the second disc (44) and setting $d_{3} \rightarrow \infty$.

${ }^{4}$ Other possible sufficient conditions can be achieved. Another intuitive condition is retrieved from disc (44) setting $d_{1}=d_{4}=1, d_{4}=0$ and $d_{3} \rightarrow \infty$ :

$$
\eta \frac{1-\alpha}{\beta}\left(1+\frac{1}{\alpha}\right)-(1-\phi)<0
$$

As (47) is a more restrictive condition than (46), we have selected this one for presentation in the main text.
} 
Proof. The initial values $\mathbf{X}(0)$ satisfy:

$$
\mathbf{X}(0)-\mathbf{X}^{*}=\sum_{i=1}^{2} \Omega_{i} \mathbf{b}_{i},
$$

where $\mathbf{b}_{i}$ are the eigenvectors corresponding to the two stable eigenvalues, and $\Omega_{i}$ are constants that can be determined. The system (48) comprises 4 equations into 5 unknowns $\left(C / K(0), r(0), g_{n}(0), \Omega_{1}(0), \Omega_{2}(0)\right)$. Equations (10), (23) and (29) may be re-written as:

$$
r(0)=\frac{(\alpha \eta)^{\frac{\eta}{1-\eta}}\left(\frac{(1-\beta-\eta)\left(\xi+\phi g_{n}(0)\right)}{\epsilon(1-\alpha) \eta}\right)^{\frac{1-\beta-\eta}{1-\eta}} n(0)^{\frac{(1-\phi)(1-\beta-\eta)+\eta\left(\frac{1-\alpha}{\alpha}\right)}{1-\eta}}}{K(0)^{\frac{1-\beta-\eta}{1-\eta}}}
$$

With this additional equation, we reach a system with 5 equations into 5 unknowns. This means that the stable variables select a specific starting point in the transition path.

This theorem establishes that there is a unique transition path to the steady-state, under some reasonable conditions. This is a result similar to that in Arnold (2006) and Eicher and Turnovsky (2001), but in a model with human capital accumulation.

\section{Calibration and Adjustment Paths}

In this section, we present calibration exercises and compute an adjustment path for a set of typical values of parameters. As in Eicher and Turnovsky (2001) and in Arnold (2006), we use calibration exercises to show that with usual calibration parameters, we reach two complex conjugate stable eigenvalues and two unstable roots, pointing out that with high probability the model transition path would be uniquely determined. For the baseline calibration in Gómez (2005:12), the sufficient condition for stability (46) is obtained for markups lower than $1.75(\alpha>0.57)$, thus for much reasonable values.

Remark 1 Experimentation with numerical values shows that there are two complex conjugate stable eigenvalues for a broad range of parameters (see Example 1 below), thus predicting oscillatory convergence to the steady-state. However, it is possible to construct counterexamples (see Examples 2 and 3 below). 
Example 1 For oscillatory transition through a stable path: with the benchmark calibration in Gómez (2005) - $\beta=0.36 ; \eta=0.36 ; \alpha=0.4 ; \xi=$ $0.05 ; \rho=0.023 ; \theta=2 ; \delta=0.1$ - and $\phi=0.4$, eigenvalues are 0.0448 ; $0.2487 ;-0.0218+0.0740 i$ and $-0.0218-0.0740 i$. For the benchmark calibration in Funke and Strulik (2000) $-\beta=0.36 ; \eta=0.36 ; \alpha=0.54 ; \xi=0.05$; $\rho=0.023 ; \theta=2 ; \delta=0.1$ - and $\phi=0.4$, eigenvalues are $0.049 ; 0.1753$; $-0.0345+0.0640 i$ and $-0.0345-0.0640 i$. For the benchmark calibration in Gómez (2005) and $\phi=0.8$, eigenvalues are $0.0477 ; 0.2216 ;-0.0171+0.0542 i$ and $-0.0171-0.0542 i$.

Example 2 For monotonous transition through a stable path: with the first calibration in Example 1 but with $\alpha=0.94$, eigenvalues are $0.0374 ; 0.0833$; -0.0651 and -0.0560 .

Example 3 For an unstable steady-state: with the first calibration in Example 1 but with $\alpha=0.20$, eigenvalues are $0.0474 ; 0.5433 ; 0.0006+0.0824 i$ and $0.0006-0.0824 i$.

As a sensitivity analysis exercise we have considered different values for the substitutability between varieties (which also governs the markup) and for the spillovers, maintaining other parameters as in the examples. While variations in the value of spillovers maintain the stability and the oscillatory pattern (holding other parameters constant), extreme markup values make the difference regarding determinacy and monoticity. For very high substitutability (low markup) $-\alpha \geq 0.94$, the balanced-growth path comes out to be determinate, but without the transition oscillatory pattern (two negative real roots). For very low substitutability - $\alpha \leq 0.2$ (high markup), the steady-state turns out to be unstable (the four roots come out to be positive). No drastic rise in the substitutability parameter $\alpha$ from the ones consider in the Examples implies that there is a positive threshold for spillovers below which monotonous transitional dynamics arise (i.e. the stable roots are real). For instance for $\alpha=0.54$ the threshold is 0.02 and for $\alpha=0.8$ the threshold is 0.22 .

In the next few lines we describe the adjustment path of an economy calibrated with the first set of values presented in Example $1 .{ }^{5}$ To integrate the fourth-order system of differential equations we use the method of backward integration described by Brunner and Strulik (2002). Figure 1 shows

\footnotetext{
${ }^{5}$ Results for adjustment paths that result from the different sets of parameter values presented above are available from the author upon request.
} 
adjustment paths of the growth rate of physical capital, the interest rate, the shares of human capital allocated to each of the sectors, the human capital and varieties growth rates and the human capital-varieties ratio. ${ }^{6}$
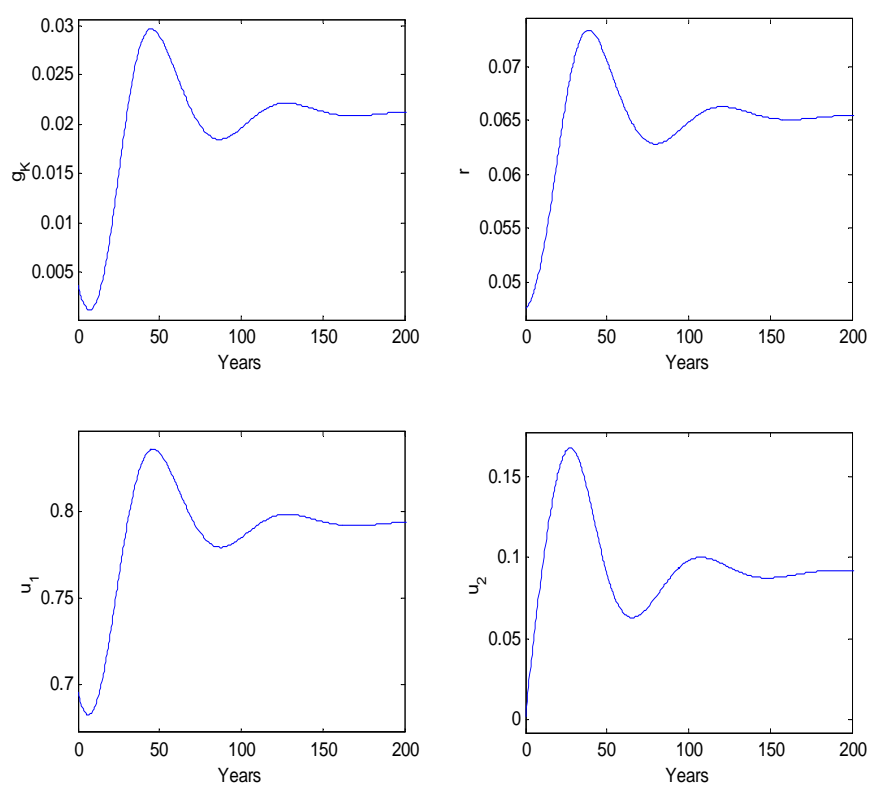

\footnotetext{
${ }^{6}$ These figures can be compared with Figure 4 in Gómez (2005).
} 

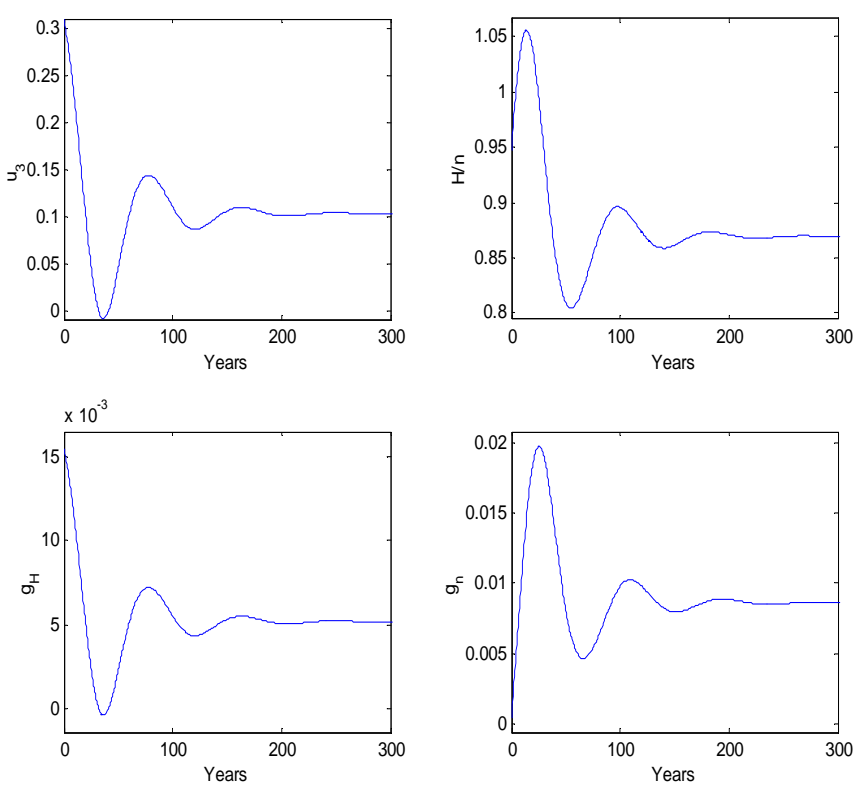

Figure 1: Transition Paths for Representative Variables

Figures show oscillatory adjustment until the steady-state. Intuitively, the presence of spillovers increases the investment in $R \& D$ and the growth rates of per capita output, when compared to Gómez's (2005) results. Overall, there is an overshooting effect at the beginning of the transition path, compared with several oscillations showed in Gómez (2005). Moreover, the presence of or increase in the spillovers parameter increases the number of years the economy takes to reach the steady-state.

\subsection{Discussion}

The model presented and studied here introduces spillovers in the R\&D process in a model which already included the most studied sources of growth: physical and human capital and the increasing number of varieties (without spillovers). Thus, the importance of this model and its features depends crucially on the evidence of the existence of spillovers in the R\&D process. In Romer's seminal model of endogenous technological change (Romer, 1990) the more resources the economy allocates to $\mathrm{R} \& \mathrm{D}$, the more it would grow (the so-called scale-effect). The evidence on the simultaneity between increasing resources in R\&D and stable TFP growth led Jones (1995) to model 
R\&D with decreasing returns to the stock of knowledge. This assumption of $0<\phi<1$ is now assumed in a stream of literature called non-scale R\&D growth models. Some studies have supported the existence of high spillovers calculating a rate of social return to $R \& D$ higher than the private one. Jones and Williams (1998), for instance, conclude that optimal R\&D expenditure is at least four times higher than decentralized spending. A fruitful literature has regressed TFP growth on R\&D stock and concluded for significant effects (Coe and Helpman, 1995). With particular interest for evaluating the empirical plausibility of our model with human capital accumulation are the extensions to the Coe and Helpman regressions provided by Engelbretch (1997) and Barrio-Castro (2002). Apart from a superior role for human capital reported in this last article, both conclude for a significant relationship between TFP and the stock of knowledge, even in the presence of human capital. Thus, the presence of spillovers in R\&D is not only at the center of recent endogenous growth theory, but has also been empirically supported.

This model also predicts features that were not addressed by the earlier ones. The Arnold/Gómez model predicts oscillatory convergence, but the condition for stability implies implausible high values for the markups. Norrbin (1993) presented markups for sectors in the USA, all below 1.7, while the condition for stability in Gómez (2005) implies a markup higher than 2. It is worth noting that in our model, for reasonable values of markups (between 1.2 and 1.4 in Norrbin, 1993), the uniquely determined oscillatory pattern through a two-dimensional stable manifold arises. For lower markup, the model continues to be stable but presents a monotonous trajectory to the steady-state. Whether the evidence calls for monotonous or oscillatory convergence is an issue under discussion. Historical evidence of the stages of development of the most developed countries seems to suggest monotonous convergence (Maddison, 2001:74). Nevertheless, if one thinks on the experience of different less developed economies in the post-war period, one can argue that modern economies grow through cycles (Fiaschi and Lavezzi, 2003, 2007). Our Figure 1 (and all paths resulting from calibration values in Example 1) resembles an initial fast-growing take-off and then a slowdown until the steady-state, similar to what was suggested by Fiaschi and Lavezzi. The Eicher and Turnovsky (2001) model also predict an overshooting of final values during the transition. The Jones/Arnold model also predicts monotonic or oscillatory convergence (but with a prevalence of monotonic convergence for most sets of parameters) and can rule out the case of unstable steady-state. This is not the case in Eicher and Turnovsky 
(2001), where instability cannot be ruled out. That article also presents a sufficient condition for stability. For high markups in our model, the steadystate is unstable. The dependence of our model properties on the markup is interesting, given Gali's (1994) contribution on the crucial effects of markups in growth models. There are interesting policy implications from the sufficient conditions stated in Lemma 3: economies with relatively high share of physical capital relative to the differentiated-goods sector share (high $\beta / \eta$ ), with relatively high markup (high $1 / \alpha$ ) and high spillovers (high $\phi$ ) tend to be unstable, thus showing diverging paths from the initial endowments.

\section{Conclusion}

The consideration of spillovers in a model with both R\&D processes and human capital accumulation has not been done previously. Nevertheless, two relevant empirical contributions (Engelbrecht, 1997 and Barrio-Castro, 2002) have proved that in empirical specifications that included human capital, R\&D spillovers were still high in value and presented high statistical significance.

We introduce R\&D spillovers into the endogenous growth model with physical capital, human capital and varieties due to Arnold (1998, 2000a). We study the steady-state and the convergence properties of the model. Furthermore, we solve it numerically to obtain a transition path to the steady-state.

The dynamics of the model are characterized by the behavior of a system of four differential equations. This article provides one more example of the analysis of a system of four ODEs in economics, and of the possible tools to implement.

The system can be either stable or unstable. Under mild conditions it proves to be characterized by a two-dimensional stable manifold and converges through a uniquely determined balanced growth path to the steadystate. Thus, when compared to the model without spillovers, their presence increases the order of differential equations system to four, also providing a two-dimensional stable manifold. This model maintains the oscillatory convergence and allows for more reasonable markup values to fulfil stability conditions. When compared with a model without human capital accumulation (Jones, 1995), the stability properties also indicate a two-dimensional

stable manifold. Two differences arise. First, we cannot rule out instability as can be done in Jones' model. Second, for most reasonable parameter sets, 
the transition to the steady-state in the model presented here is oscillatory and not monotonous, as it is in the Jones model.

We found a crucial effect of the markup distortion in the convergence properties of the model. For high markups the steady-state is unstable. For intermediate values, the steady-state is stable and the transition oscillatory. For low markups, the steady-state is stable and the transition monotonous.

\section{References}

[1] Arnold, L. (2006), "The Jones R\&D Growth Model: Comment on Stability", Review of Economic Dynamics, 9(1), 143-152.

[2] Arnold, L. (2000a), "Endogenous Growth with Physical capital, Human capital and Product variety: A comment", European Economic Review, 44, 1599-1605.

[3] Arnold, L. (2000b), "Endogenous Technological Change: A note on stability", Economic Theory, 16, 219-226.

[4] Arnold, L. (2000c), "Stability of the Market Equilibrium in Romer's Model of Endogenous Technological Change: A complete characterization", Journal of Macroeconomics, 22(1), 69-84.

[5] Arnold, L. (1998), "Growth, welfare and trade in an integrated model of human-capital accumulation and research", Journal of Macroeconomics, 20(1), 81-105.

[6] Barrio-Castro, T., López-Bazo, E. and Serrano-Domingo, G. (2002), "New Evidence on international R\&D spillovers, human capital and productivity in the OECD", Economics Letters, 77, 41-45.

[7] Benhabib, J. and R. Perli (1994), "Uniqueness and Indeterminacy: On the dynamics of Endogenous Growth", Journal of Economic Theory, $63,113-142$.

[8] Brunner, M. and H. Strulik (2002), "Solution of Perfect Foresight Saddlepoint Problems: A simple method and aplications", Journal of Economic Dynamics and Control, 25(5):737-753, May.

[9] Coe, D. and E. Helpman (1995), "International R\&D spillovers", European Economic Review 39, 859-887. 
[10] Eicher, T. and S. Turnovsky (2001), "Transitional Dynamics in a TwoSector non-scale Growth Model", Journal of Economic Dynamics and Control, 25, 85-113.

[11] Engelbrecht, H. (1997), "International R\&D spillovers, human capital and productibity in OECD countries: An Empirical investigation", European Economic Review, 41, 1479-1488.

[12] Fiachi, D. and M. Lavezzi (2003), "Distribution Dynamics and Nonlinear Growth", Journal of Economic Growth, 8, 379-401.

[13] Fiachi, D. and M. Lavezzi (2007), "Nonlinear Economic Growth and Cross-Country Evidence", Journal of Economic Development, 84(1), 271-290.

[14] Funke, M. and H. Strulik (2000), "On Endogenous growth with physical capital, human capital and product variety", European Economic Review, 44, 491-515.

[15] Gali, J. (1994), "Monopolistic Competition, Endogenous Markups and Growth", European Economic Review 38, 748-756.

[16] Gómez, M. (2005), "Transitional Dynamics in an Endogenous growth model with physical capital, human capital and R\&D", Studies in Nonlinear Dynamics 8 Econometrics, 9, 1, Article 5.

[17] Griliches, Z. (1992), "The search for R\&D Spillovers", Scandinavian Journal of Economics, 94 (Supplement), 29-47.

[18] Horn, R. and C. Johnson (1985), Matrix Analysis, Cambridge University Press, Cambridge, UK.

[19] Jones, C. (1995), "R\&D-based models of endogenous growth", Journal of Political Economy, vol. 103, n.4, 759-584.

[20] Ladrón-de-Guevara, S. Ortigueira and M. Santos (1997), "Equilibrium Dynamics in Two-Sector Models of Endogenous Growth", Journal of Economic Dynamics and Control, 21, 115-143.

[21] Maddison, A. (2001), The World Economy, a millenial perspective, Development Center Studies, OECD, Paris. 
[22] Norrbin, S. (1993), "The Relation between Price and Marginal Cost in U.S. Industry: A contradiction", Journal of Political Economy, vol. 101, 6, pp. 1149-1164.

[23] Porter, M. and S. Stern (2000), Measuring the 'Ideas' Production Function: evidence from international patent output, NBER Working Paper 7891.

[24] Romer, P. (1990), "Endogenous Technological Change", Journal of Political Economy, 98, S1971-2102. 